\title{
Significance of data analysis in the quality control of prenatal screening for Down syndrome
}

\author{
YAHONG LI $^{1 *}$, XIAOJUAN ZHANG ${ }^{1 *}$, DONGYANG HONG ${ }^{1}$, XIANWEI GUAN ${ }^{1}$, \\ SHAOLEI LV ${ }^{2}$, YUN SUN ${ }^{1}$ and TAO JIANG ${ }^{1}$ \\ ${ }^{1}$ Center of Prenatal Diagnosis, The Affiliated Obstetrics and Gynecology Hospital of \\ Nanjing Medical University, Nanjing Maternity and Child Health Care Hospital, Nanjing, Jiangsu 210004; \\ ${ }^{2}$ Zhejiang Biosan Biochemical Technologies Co., Ltd., Hangzhou, Zhejiang 310000, P.R. China
}

Received November 1, 2017; Accepted February 9, 2018

DOI: $10.3892 /$ br.2018.1078

\begin{abstract}
Dual detection of $\alpha$-fetoprotein (AFP) and free $\beta$-human chorionic gonadotropin $(\beta-\mathrm{HCG})$ is a common screening method for Down syndrome in the second trimester and its efficacy is assessed by false-positive rate (FPR). The present study aimed to investigate the effects of the bias in median multiple of the median (mMoM) values of AFP and free $\beta-\mathrm{HCG}$ on FPR. The bias in $\mathrm{mMoM}$ values of AFP and free $\beta-\mathrm{HCG}$ and the bias in $\mathrm{mMoM}$ values under different gestational ages and weight groups were analyzed. Median equations were adjusted, and medians in LifeCycle software were replaced by local medians. Following two adjustments of the median equations, all indices including FPR, mMoM values of markers and $\mathrm{mMoM}$ values under different gestational ages and weight groups generally reached an ideal state. In conclusion, abnormal bias in mMoM values may prompt aberrant application of median equations, and regular monitoring of these indicators may be important for quality control in prenatal screening.
\end{abstract}

\section{Introduction}

Down syndrome is considered to be the most common disease among prenatal chromosome abnormalities (1). Prenatal serological screening is an effective method for decreasing the birth of prevalence of children with Down syndrome, and adequate quality control of the screening is important to maintain its efficiency (2). Prenatal screening for Down syndrome involves coordination of multiple clinical sectors, and its efficiency depends on a variety of factors, including clinical

Correspondence to: Dr Tao Jiang, Center of Prenatal Diagnosis, The Affiliated Obstetrics and Gynecology Hospital of Nanjing Medical University, Nanjing Maternity and Child Health Care Hospital, 123 Tianfei Road, Nanjing, Jiangsu 210004, P.R. China E-mail: jiangzhang784@163.com

*Contributed equally

Key words: quality control, prenatal screening, Down syndrome data collection, serum and treatment, detection method and risk calculation. Due to numerous and uncertain factors, it is difficult to monitor the complete process of prenatal screening for Down syndrome (3-5).

Second-trimester biochemical screening methods include the detection of dual serological indicators, namely $\alpha$-fetoprotein (AFP) combined with total human chorionic gonadotropin (HCG) or free $\beta$-HCG $(6,7)$, a triple test for AFP, unconjugated estriol (uE3) and HCG (8), and a quadruple test for AFP, HCG (total or free $\beta$-), uE3 and inhibin-A (9). The median multiple of the median (mMoM) value for different markers is considered to be a standard metric as it responds to the quality of data effectively. The detection rate (DR) and false positive rate (FPR) are also considered to be effective evaluation indices of screening efficiency, though they may be affected by the bias in MoM values of distinct markers (8). Nix et al (10) identified that if there was a bias of $10 \%$ in MoM value for individual markers, there would be a 4-fold increase in risk of Down syndrome determined via the triple test. For instance, if a risk was 1/1,000 and all three markers had a $10 \%$ bias in MoM values towards Down syndrome, the risk would be $1 / 361$. The risk threshold changed from $1 / 250$ to $1 / 692.5$, meanwhile FPR and DR increased simultaneously. If the risk threshold was $1 / 250$, FPR and DR were 5.8 and $75.6 \%$, respectively. Considering a risk threshold of $1 / 692.5$, FPR would be $13.5 \%$ and DR would be $86.9 \%$, thus increasing FPR by $7.7 \%$ and DR by $11.3 \%$. These data indicated that even a $5 \%$ bias in a single marker would lead to up to a $2 \%$ change in FPR (10). Increased FPR is a source of mental stress for pregnant women, as well as being wasteful in terms of medical resources and cost. Therefore, it is important to observe FPR and $\mathrm{mMoM}$ values regularly to maintain effective screening quality. The present study aimed to evaluate the practical bias phenomenon in mMoM values at the Nanjing Maternity and Child Health Care Hospital (Nanjing, China), and to determine strategies of reducing the bias in order to improve screening and provide improved quality control management.

\section{Materials and methods}

Population selection. A total of 109,952 female subjects (mean age, 28.20 \pm 3.32 ) with singleton pregnancies, who accepted the 
second trimester screening for Down syndrome at $15^{+0}$ and $20^{+6}$ weeks at the Nanjing Maternity and Child Health Care Hospital from January 2014 to December 2016, were included in the current study. Ethical approval following review of the study protocols was obtained from the Medical Ethics Committee of Nanjing Maternity and Child Health Care Hospital, and written informed consent was obtained from all participants following full disclosure of the study procedures.

Instruments and reagents. AFP and free $\beta-\mathrm{HCG}$ in serum were detected with a 1235 automatic fluorescence immunoassay analyzer (PerkinElmer, Inc., Waltham, MA, USA) using a Wallac Auto DELFIA ${ }^{\circledR}$ hAFP/Free hCG $\beta$ Dual kit (PerkinElmer, Inc.). Control serum (batch nos. 20140301, 20150401 and 20151101) with high, medium and low concentrations (Zhejiang Biosan Biochemical Technologies Co., Ltd., Zhejiang, China) was assayed under the same condition to verify the reliability of the experiment. The ranges of low, medium and high for each batch were obtained from a sufficient number of tests following on from the previous quality control batch.

mMoM monitoring of serum biomarkers AFP and free $\beta$-HCG. mMoM value fluctuating between 0.95 and 1.05 was considered to be acceptable, and values beyond this range indicated that certain factors may be influencing the bias of screening and influence DR and FPR (11).

Adjustment of gestational age and weight median equations. In general, FPR of $~ 5 \%$ with slight fluctuation was considered normal (12); if FPR was markedly higher or lower the screening was deemed unsatisfactory. An increase in FPR may ultimately cause an increase in the number of invasive prenatal examinations, in the psychological burden to pregnant women and in pressure to medical capacity. From May 2015 to August 2015, it was identified that the FPR was high $(>6 \%)$. Subsequently, mMoM values obtained each month between January 2014 and August 2015 for individual markers were retrospectively analyzed. Following the identification of continuous bias in mMoM values of AFP and free $\beta$-HCG towards Down syndrome, their mMoM values were observed between different gestational ages and weight groups, as listed in Table I. Initially, only gestational age and weight median equations for AFP were adjusted, as well as the weight median equation for free $\beta$-HCG based on the study cohort data from January 2014 to August 2015. Medians embedded in LifeCycle software version 4.0 (PerkinElmer, Inc.) were replaced by medians calculated according to the local data from January 2014 to August 2015. However, it was identified that $\mathrm{mMoM}$ values of free $\beta$-HCG in the next months (September 2015 to March 2016) had increased bias compared with the values from the preceding months. Thus, both gestational and weight median equations for free $\beta$-HCG were adjusted using the study cohort data from January 2014 to December 2015. The medians embedded in the LifeCycle software were then replaced by the medians calculated over this time period. The data from January 2014 to December 2016 was retrospectively analyzed applying the adjusted median equations. Whether the adjustment was appropriate was verified by comparing FPR, mMoM values of AFP and free
Table I. Numbers of patients in different gestational age and weight groups.

\begin{tabular}{lrcr}
\hline Gestational age (weeks) & $\mathrm{n}$ & Weight $(\mathrm{kg})$ & \multicolumn{1}{c}{$\mathrm{n}$} \\
\hline $15^{+0}-15^{+6}$ & 4,707 & $40-50$ & 9,805 \\
$16^{+0}-16^{+6}$ & 20,067 & $51-55$ & 16,582 \\
$17^{+0}-17^{+6}$ & 26,177 & $56-60$ & 14,946 \\
$18^{+0}-18^{+6}$ & 9,314 & $61-65$ & 9,590 \\
$19^{+0}-19^{+6}$ & 3,128 & $66-70$ & 7,822 \\
$20^{+0}-20^{+6}$ & 1,013 & $71-75$ & 2,427 \\
& & $76-80$ & 2,031 \\
& & $81-85$ & 516 \\
& & $85-90$ & 441 \\
& & $>90$ & 246 \\
\hline
\end{tabular}

$\beta$-HCG markers, and mMoM values of AFP and free $\beta$-HCG under different gestational ages and different weight groups prior to and following the adjustments of the median equations.

Cumulative sum control (CUSUM) chart analysis. A CUSUM chart accumulates and magnifies the bias occurring during a detection process. When the mean value of the measured results coincides with the expected value, the cumulative trend is parallel to the time axis. When the deviation of the mean value from the expected value is positive, the cumulative trend inclines upwards and vice versa. The larger the difference between the measured result and the expected value, the greater the accumulation and the steeper the inclination of the graph. By observing the change in the slope of the curve, the change and the starting point of the change can be identified $(13,14)$. The current study analyzed whether the change was consistent with mMoM values of AFP and free $\beta-\mathrm{HCG}$, and its function in quality control was evaluated.

Statistical analysis. SPSS 17.0 software (SPSS, Inc., Chicago, IL, USA) was used for statistical analysis. Independent samples t-tests were used for analysis of data significance, Data was presented as the mean \pm two standard deviations and $\mathrm{P}<0.05$ was considered to indicate a statistically significant difference.

\section{Results}

FPR increase and bias in $M M$ M $M$ values of AFP and free $\beta-H C G$ markers. FPR is a quality control aspect of serological screening, which in the current study was identified to be $\sim 5 \%$

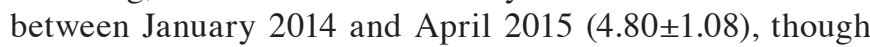
was comparatively higher from May 2015 (5.74 $\pm 1.20 ; \mathrm{P}>0.05$; Fig. 1A). Subsequently, the detection precision and accuracy factors that may lead to an increased FPR, including serum processing and testing, and gestational age projections, were excluded, and the mMoM values of AFP and free $\beta$-HCG markers were retrospectively analyzed. From March to October 2014 and March to August 2015, there was continuous bias. $\mathrm{mMoM}$ values of AFP fluctuated in the range of 0.95-1.00 (Fig. 1B); and mMoM values of free $\beta$-HCG fluctuated in the range of 0.95-1.05 from January 2014 to April $2015(0.99 \pm 0.04)$, 
A

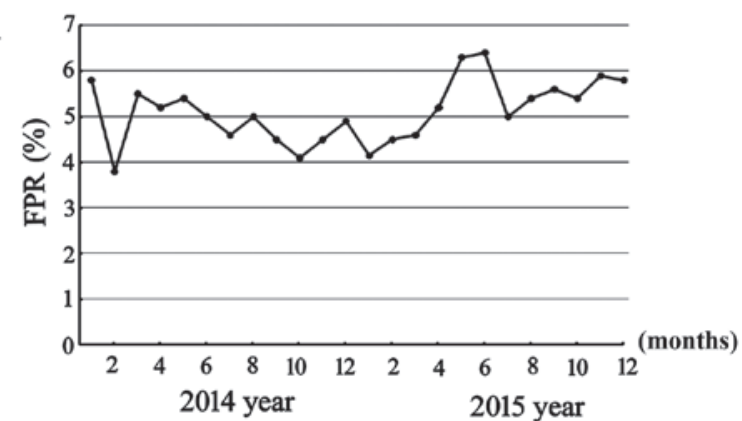

B
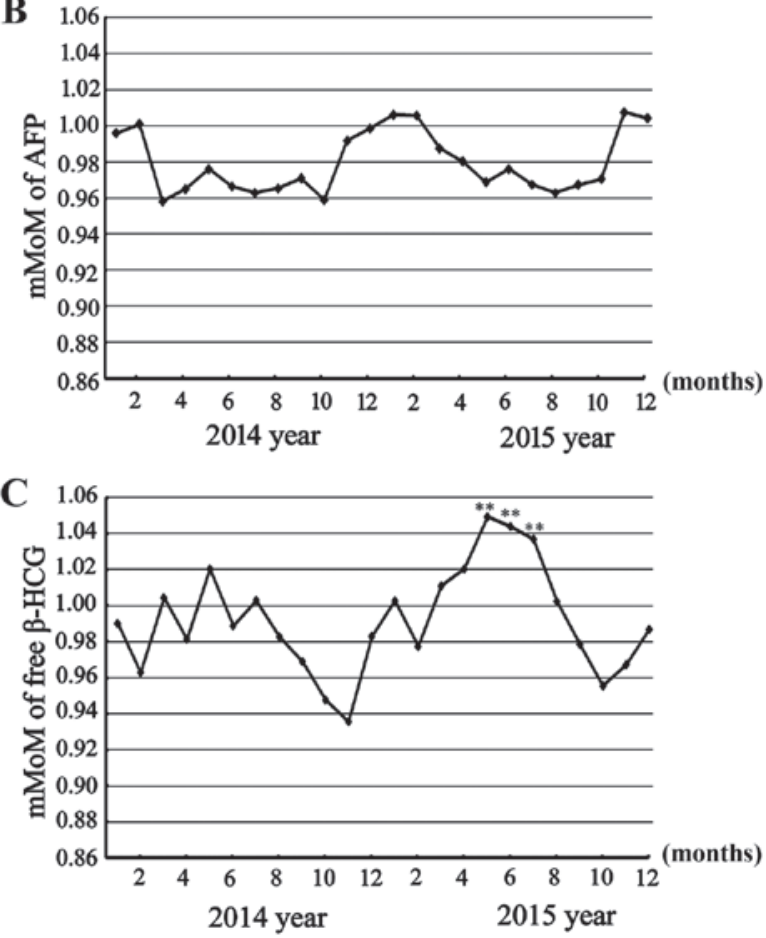

Figure 1. Monthly FPR and mMoM values of AFP and free $\beta$-HCG markers. (A) FPR from January 2014 to December 2015: A sharp increase was observed from May 2015. (B) mMoM values of AFP marker from January 2014 to December 2015: There was two periods during which $\mathrm{mMoM}$ values were continuously maintained in the range of $0.95-1.00$. (C) $\mathrm{mMoM}$ values of free $\beta$-HCG marker from January 2014 to December 2015: mMoM values abnormally increased from May 2015 to July $2015,{ }^{* *} \mathrm{P}<0.01$ vs. mMoM values of free $\beta$-HCG marker from January 2014 to April 2015. FPR, false positive rate; $\mathrm{mMoM}$, median multiple of the median; AFP, $\alpha$-fetoprotein; free $\beta$-HCG, free $\beta$-human chorionic gonadotropin.

and rarely outside this range, but it was persistently above 1.0 from May 2015 to July $2015(1.04 \pm 0.02 ; \mathrm{P}<0.01)$, thus indicating significant bias towards Down syndrome (Fig. 1C). These biases may have been the cause of high FPR.

Influences of gestational age and weight median equations on $m M o M$ values. Following the identification of bias in $\mathrm{mMoM}$ values of AFP and free $\beta$-HCG markers, $\mathrm{mMoM}$ values of AFP and free $\beta$-HCG were retrospectively analyzed under different gestational ages and weight groups. This identified bias in the mMoM values of AFP under the different gestational ages and weight groups. The preliminary gestational age median equation for AFP was not calibrated for gestational ages from 17 weeks (119 days) to 20 weeks (140 days), as mMoM values fluctuated in the range 0.95-1.0 and were sometimes <0.95; therefore, the median equation of gestational age was adjusted from the original $y=10^{\wedge}\left(0.514018+0.00872271^{*} \mathrm{GA}+0.0000\right.$ $\left.00182258^{*} \mathrm{GA}^{\wedge} 2\right)$ to $\mathrm{y}=10^{\wedge}\left(1.77831-0.0101412^{*} \mathrm{GA}+0.00007\right.$ $\left.16186 * \mathrm{GA}^{\wedge} 2\right)$. When the new median equation was applied, $\mathrm{mMoM}$ values of AFP under different gestational ages from January 2014 to May 2016, or data from September 2015 to March 2016 under different gestational ages resulted in reliable curves, with $\mathrm{mMoM}$ values fluctuating from 0.95 to 1.05 randomly (Fig. 2A). The gestational age median equation of free $\beta$-HCG was not changed at this time, as there was no apparent unsuitability using the median equation $\mathrm{y}=10^{\wedge}(-4.53$ $412+0.270717 * \mathrm{GA}-0.00398489 * \mathrm{GA}^{\wedge} 2+0.0000237835^{*} \mathrm{GA}^{\wedge} 3-$ $\left.0.0000000510437 * \mathrm{GA}^{\wedge} 4\right)$ provided by the LifeCycle software (Fig. 2B). For the weight median equation, it was identified that both calibrations were inadequate (Table II); the mMoM values of AFP and free $\beta$-HCG under different weight groups were biased markedly towards Down syndrome; therefore, the weight median equation of AFP was changed from $\mathrm{y}=0.43391+37.643 /$ weight to $\mathrm{y}=0.276478+40.9054 /$ weight, and the weight median equation of free $\beta$-HCG was changed from $\mathrm{y}=10^{\wedge}\left(0.276-0.004^{*}\right.$ weight $)$ to $\mathrm{y}=10^{\wedge}(0.495775-0.0107$ $661^{*}$ weight $+0.000036501 *$ weigh $^{\wedge} \wedge$ ), based on the data from January 2014 to December 2015. Following the change, mMoM values of AFP under different weight groups came to an ideal distribution state, generally fluctuating in the range of 0.95 to 1.05 , though some were over the range (Table II). However, correcting the body weight median equation and medians in LifeCycle software for free $\beta$-HCG did not achieve the desired effect. From September 2015 to March 2016 following this adjustment, FPR was sustained at a high level (Fig. 3A), and while $\mathrm{mMoM}$ values of AFP marker fluctuated randomly in the range $0.95-1.05$ (Fig. 3B), greater deviation in $\mathrm{mMoM}$ values of free $\beta$-HCG occurred under different gestational ages (Fig. 2B) and different weight groups (Table II). Meanwhile, $\mathrm{mMoM}$ values of the $\beta$-HCG marker sustained bias, fluctuating from 1.06 to 1.20 from September 2015 to May 2016 after using the adjusted local median equation. When applying the adjusted median equation to retrospectively analyze the data from January 2014 to December 2016, the same bias was observed (Fig. 3C). It was determined that by only changing the median equation of weight of free $\beta$-HCG in September 2015, bias of free $\beta$-HCG also existed, and thus the weight median equation was adjusted as $\mathrm{y}=2.416-0.03435^{*}$ weight +0.0001674 $*_{w}$ eight ${ }^{\wedge} 2$, and the gestational age median equation as $\mathrm{y}=10^{\wedge}$ (3.59566-0.030088*GA+0.0000794176*GA^2) in March 2016 according to data from January 2014 to December 2015, and the medians of the software were also adjusted according to data from January 2014 to December 2015. Subsequently, the mMoM values of free $\beta$-HCG marker came to an ideal distribution state (Fig. 3D), fluctuating between $\sim 0.95-1.05$, and even the mMoM values of free $\beta$-HCG under different gestational ages (Fig. 2B) and different weights (Table II) returned to normal range after these measures. Additionally, the FPR from April 2016 to December 2016 returned to an acceptable range (Fig. 3A).

Significance of CUSUM chart in screening quality control. CUSUM charts were considered effective for responding to quality control, as they changed consistently with the adjustments performed. From June 2014 to December 2014, the slope of the curve in the CUSUM charts of AFP (Fig. 4A) continued 
Table II. mMoM values of AFP and free $\beta$-HCG prior to and following weight equation adjustment.

\begin{tabular}{|c|c|c|c|c|c|}
\hline \multirow[b]{2}{*}{$\begin{array}{l}\text { Weight } \\
\text { group }(\mathrm{kg})\end{array}$} & \multicolumn{2}{|r|}{ AFP } & \multicolumn{3}{|c|}{ Free $\beta$-HCG } \\
\hline & $\begin{array}{l}\text { mMoM values } \\
\text { given by } \\
\text { LifeCycle } \\
\text { software }\end{array}$ & $\begin{array}{l}\text { mMoM values obtained } \\
\text { following weight } \\
\text { equation adjustment } \\
\text { in September } 2015\end{array}$ & $\begin{array}{l}\text { mMoM values } \\
\text { given by } \\
\text { LifeCycle } \\
\text { software }\end{array}$ & $\begin{array}{c}\text { mMoM values } \\
\text { calculated by applying } \\
\text { equation adjusted } \\
\text { in September } 2015\end{array}$ & $\begin{array}{l}\text { mMoM values } \\
\text { calculated by applying } \\
\text { equation adjusted } \\
\text { in March } 2016\end{array}$ \\
\hline $40-50$ & 1.00 & 1.00 & 1.05 & 1.07 & 0.99 \\
\hline $51-55$ & 0.99 & 1.00 & 1.02 & 1.08 & 1.00 \\
\hline $56-60$ & 0.97 & 1.00 & 0.99 & 1.11 & 1.01 \\
\hline 61-65 & 0.96 & 1.00 & 0.95 & 1.08 & 0.99 \\
\hline $66-70$ & 0.96 & 0.99 & 0.93 & 1.08 & 1.00 \\
\hline 71-75 & 0.94 & 0.97 & 0.92 & 1.06 & 1.00 \\
\hline $76-80$ & 0.94 & 1.00 & 0.92 & 1.13 & 1.01 \\
\hline $81-85$ & 0.93 & 1.01 & 0.91 & 1.04 & 0.99 \\
\hline $86-90$ & 0.95 & 0.99 & 0.86 & 1.18 & 0.98 \\
\hline$>90$ & 0.92 & 1.03 & 0.89 & 1.20 & 1.01 \\
\hline
\end{tabular}

mMoM, median multiple of the median; AFP, $\alpha$-fetoprotein; free $\beta$-HCG, free $\beta$-human chorionic gonadotropin.
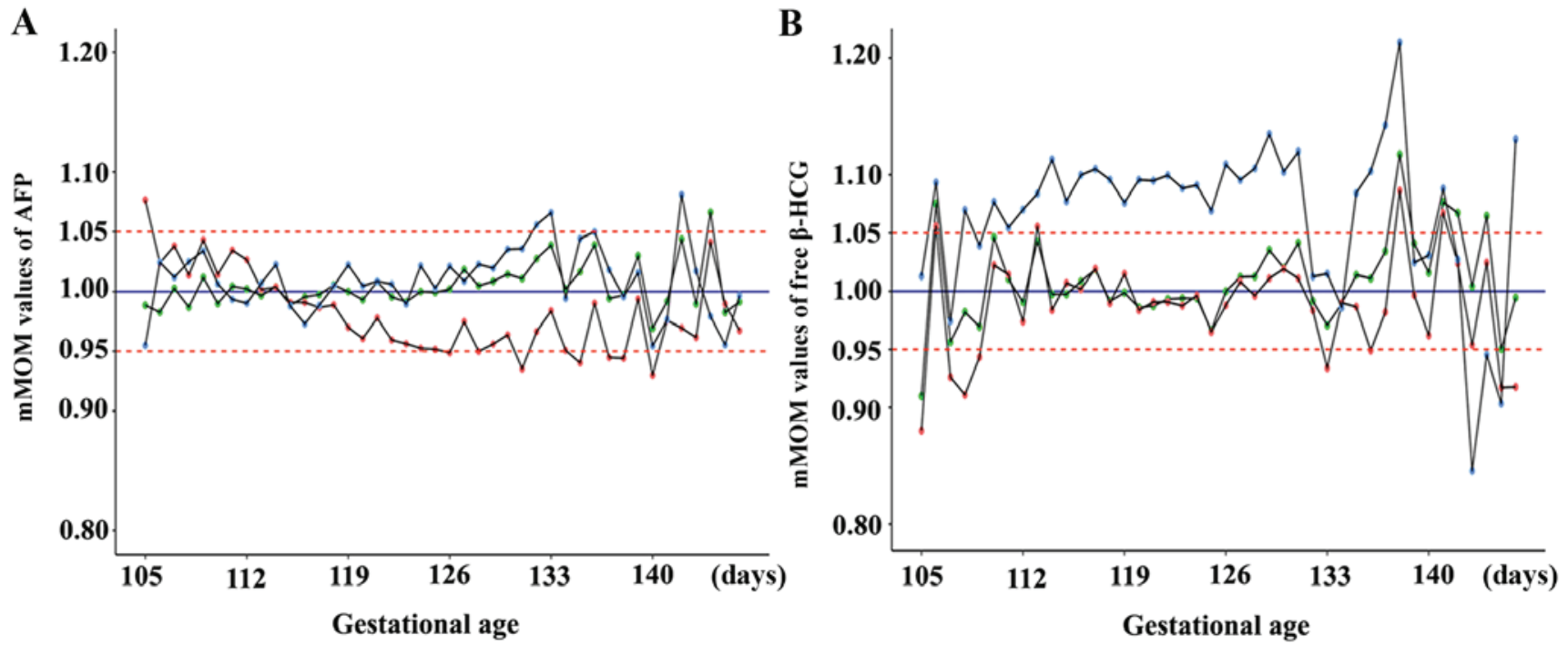

Figure 2. mMoM values of AFP and free $\beta$-HCG under different gestational ages analyzed by applying distinct median equations. (A) mMoM values of AFP under different gestational ages: Bias in mMoM values from January 2014 to July 2015 could be identified when using the median equation embedded in LifeCycle software as indicated by the red-dot polyline; if the gestational age median equation was adjusted based on local data from January 2014 to August 2015, and the new gestational age median equation was applied to retrospectively analyze data from September 2015 to March 2016 or data from January 2014 to May 2016, as indicated by the blue-dot and green-dot polyline, respectively, mMoM values of AFP fluctuated in the range of 0.95-1.05, with occasional fluctuation beyond this range. (B) mMoM values of free $\beta$-HCG under different gestational ages: The original gestational age median equation embedded in LifeCycle software was appropriate from January 2014 to July 2015 before the median equation of body weight was modified, as indicated by the red-dot polyline, but mMoM values had more bias, almost above 1.05 from September 2015 to March 2016 after adjusting the weight median equation, as indicated by the blue-dot polyline; when gestational age and weight median equations were adjusted together in March 2016, mMoM values from January 2014 to May 2016 distributed reasonably if the new gestational age median equation was used, as indicated by the green-dot polyline. mMoM, median multiple of the median; AFP, $\alpha$-fetoprotein; free $\beta$-HCG, free $\beta$-human chorionic gonadotropin.

to decline, and mMoM values of AFP remained low (Fig. 1B). Due to the lack of data for the CUSUM chart at that time, the corresponding response was not determined. After this time, the slope of the curve underwent positive and negative fluctuations, and continuous increases or decreases were not apparent. After December 2015, the curve became relatively proximal to the horizontal line, indicating that the detection of AFP was stable and the adjustment of the relevant median equation was effective (Fig. 4A). A different phenomenon was observed for free $\beta$-HCG (Fig. 4B). The curve was relatively close to the horizontal line from June 2014 to May 2015, but the slope of the curve continued upward after May 2015, indicating that some significant factor may have lead to the increase in free $\beta$-HCG while FPR increased and bias in 
A

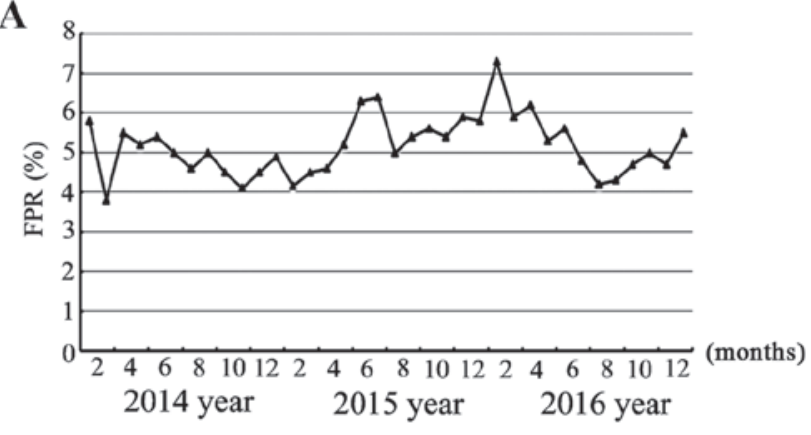

C

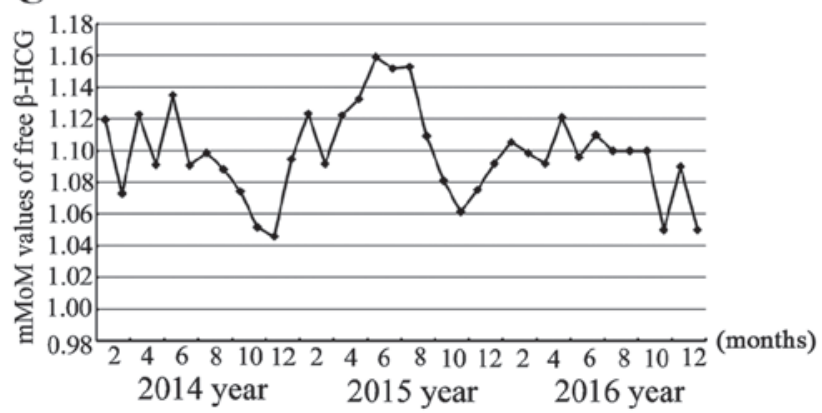

B

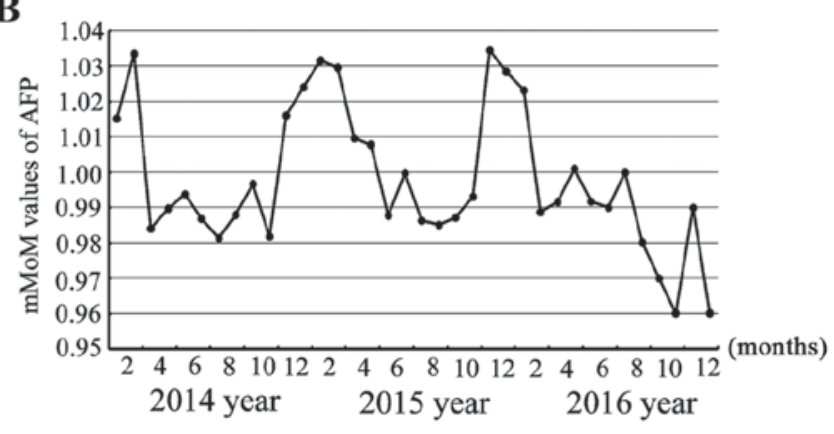

D

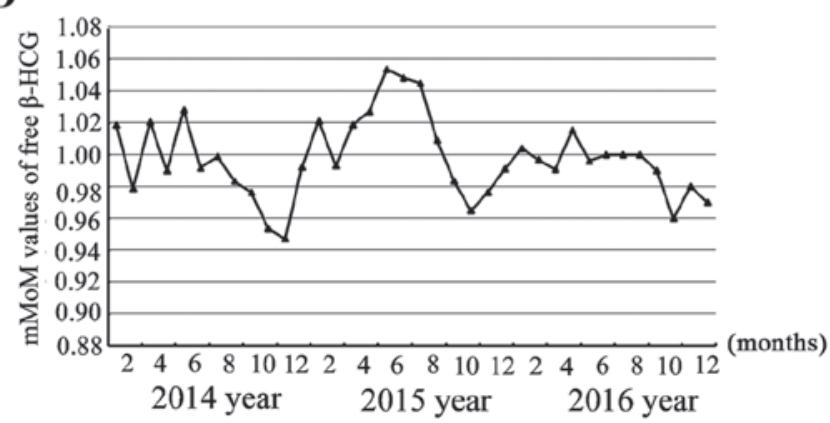

Figure 3. FPR and mMoM values of AFP and free $\beta$-HCG markers following the adjustment of median equations. (A) Monthly FPR; (B) mMoM values of AFP marker from January 2014 to December 2016 analyzed by new gestational age and weight median equations fluctuated randomly in the range of $0.95-1.05$; (C) $\mathrm{mMoM}$ values of free $\beta$-HCG from January 2014 to December 2016 analyzed by the weight median equation obtained in September 2015 exhibited continued bias towards Down syndrome; (D) mMoM values of free $\beta$-HCG from January 2014 to December 2016 analyzed by the weight median equation obtained in March 2016 fluctuated randomly in the range of 0.95-1.05. FPR, false positive rate; mMoM, median multiple of the median; AFP, $\alpha$-fetoprotein; free $\beta$-HCG, free $\beta$-human chorionic gonadotropin.

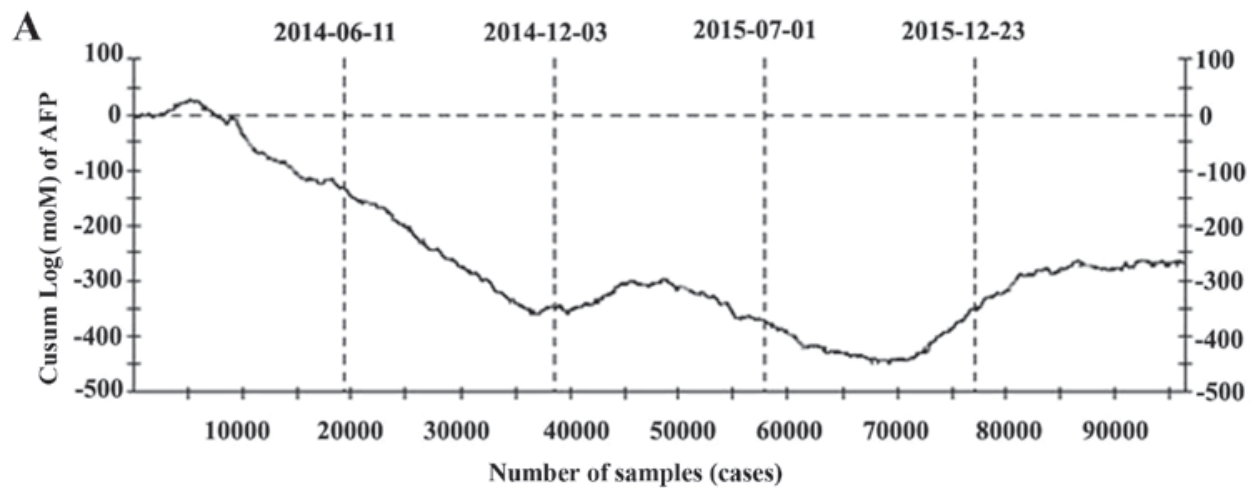

\section{B}

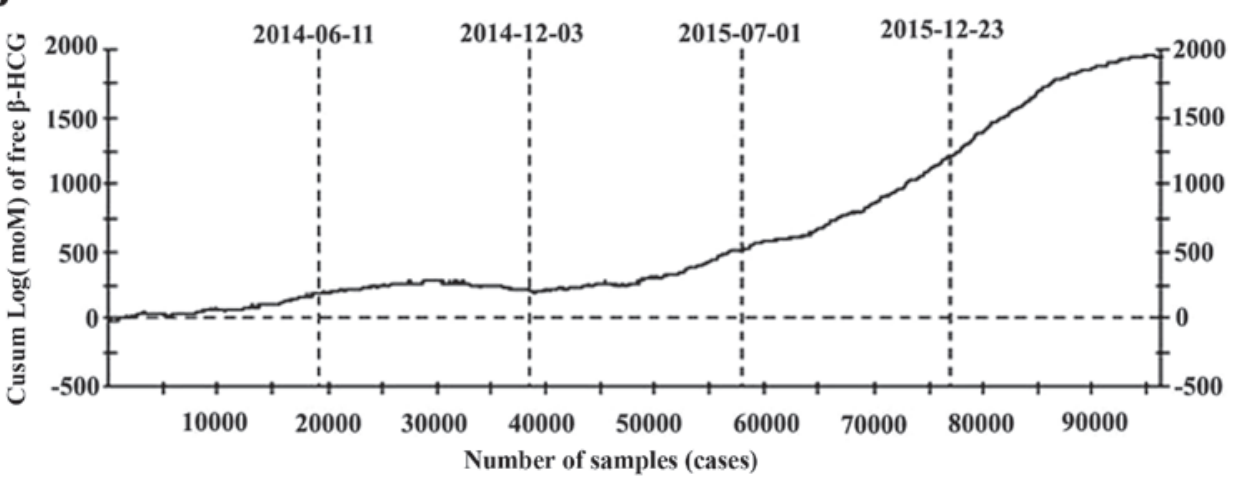

Figure 4. CUSUM charts of AFP and free $\beta$-HCG. (A) CUSUM chart of AFP from January 2014 to May 2016; (B) CUSUM chart of free $\beta$-HCG from January 2014 to May 2016. CUSUM, cumulative sum control; AFP, $\alpha$-fetoprotein; free $\beta$-HCG, free $\beta$-human chorionic gonadotropin; mMoM, median multiple of the median.

mMoM values appeared. The slope of the curve was positive from September 2015 to March 2016 after adjusting the weight median equation until the second adjustment. This phenomenon indicated that the first adjustment was inappropriate. 


\section{Discussion}

Screening efficiency is represented by both DR and FPR. Prenatal serological screening may result in false negatives, which can not be determined until after childbirth, and thus regarding quality control in prenatal screening, DR seems inadequate for immediate quality control. By contrast, FPR reflects screening quality immediately, and thus may be considered more meaningful in quality control. In the current study, a significantly elevated FPR was first identified, after which the factors that may have caused this were analyzed, including bias in the mMoM values of AFP and free $\beta$-HCG markers, and in the mMoM values of AFP and free $\beta$-HCG under different gestational ages and weight groups. The present results were similar to those reported by Nix et al (10), in that a rise in FPR and bias in $\mathrm{mMoM}$ values AFP and free $\beta$-HCG toward Down's syndrome were identified. However, an increase in FPR may increase the number of invasive prenatal examinations, the psychological burden to pregnant women and medical pressure, and thus corresponding assessments were performed. The median equations of gestational age and weight of AFP and the weight median equation of free $\beta-H C G$ were modified based on local data, while the gestational age median equation of free $\beta$-HCG was not adjusted in the first instance. In the follow-up work, FPR was markedly increased and abnormally high fluctuations in $\mathrm{mMoM}$ values of free $\beta$-HCG were observed. After a half-year period of observation, and following the exclusion of all possible factors that may cause the fluctuation, gestational age and weight median equations for free $\beta$-HCG were modified together. All indices including FPR, mMoM values of markers and $\mathrm{mMoM}$ values under different gestational ages and weight groups generally reached an ideal state. The corresponding results indicated that gestational age and weight median equations must be adjusted together and not alone.

Additionally in the present study, the significant changes in FPR and mMoM values indicated that there maybe some factor that was influencing screening. Therefore, risk assessment should be performed if there are no clear influencing factors from the start of screening to the laboratory analysis. For prenatal screening and screening management, screening data analysis is important for the quality control of the screening system. Firstly, by focusing on abnormal changes in screening FPR and mMoM values of markers, bias maybe identified immediately, which will aid to find the confounding factor at the earliest opportunity. Secondly, analysis based on local mMoM values was the basis for the modification of the median equation. Ethnic, geographical and other factors may lead to differences in mMoM values of serological indicators; therefore, establishing gestational age and weight median equations by the median acquired from normal pregnant women in the local region is important. However, how to modify the median equation is not simple and must be based on comprehensive data, accurate laboratory test results and reliable risk assessment. Thus, medians must be obtained under strict quality control. Thirdly, monitoring of all indices including FPR, mMoM values of markers and $\mathrm{mMoM}$ values under different gestational ages and weight groups is a reliable method for laboratory quality evaluation. According to this method, an independently standardized data audit method maybe established to determine a unified quality assessment method in domestic screening agencies (11). Finally, FPR and $\mathrm{mMoM}$ values may be applied to demonstrate a stable screening efficiency of the screening system, and horizontal quality evaluation could be performed among screening agencies. Taken together, the present data analysis techniques may make the screening more reliable in routine clinical together practice in the future.

\section{Acknowledgements}

Not applicable.

\section{Funding}

This study was supported by the National Natural Science Foundation of China (grant nos. 81541064 and 81671475).

\section{Availability of data and materials}

All data generated or analyzed during this study are included in this published article.

\section{Authors' contributions}

YL and XZ made substantial contributions to conception, design and involved in drafting the manuscript; $\mathrm{DH}$ and the first two authors were responsible for sample testing and Down's syndrome risk calculation; XG was responsible for collecting information; YS and all of the above authors were participated in patient informed consent; TJ was responsible for guidance and made sure the work went well. All authors read and approved the final manuscript.

\section{Ethics approval and consent to participate}

This study was approved by Medical Ethics Committee of Nanjing Maternity and Child Health Care Hospital (Nanjing, China). All pregnant women participating in the study signed written informed consent following full disclosure of the study procedures.

\section{Consent for publication}

All pregnant women participating in the study signed written informed consent permitting the publication of relevant data on the terms of data anonymization.

\section{Competing interests}

SL is an employee of Zhejiang Biosan Biochemical Technologies Co., Ltd. All other authors declare that they have no competing interests.

\section{References}

1. Smith M and Visootsak J: Noninvasive screening tools for Down syndrome: A review. Int J Womens Health 5: 125-131, 2013.

2. Duan Y, Li Y and Xue Q: Serological prenatal screening and diagnosis for Down syndrome. Clin Exp Obstet Gynecol 41: 572-574, 2014. 
3. Huang J, Chen Y and Pong RW: Factors influencing prenatal screening for Down's syndrome: Evidence from Zhejiang (China). Asia Pac J Public Health 27: NP1288-NP1297, 2015.

4. Sirichotiyakul S, Luewan S, Sekararith R and Tongsong T: False positive rate of serum markers for Down syndrome screening. Does transportation have any effect? J Med Assoc Thai 95: $152-155,2012$.

5. Wald NJ, Barnes IM, Birger R and Huttly W: Effect on Down syndrome screening performance of adjusting for marker levels in a previous pregnancy. Prenat Diagn 26: 539-544, 2006.

6. Androutsopoulos G, Gkogkos P and Decavalas G: Mid-trimester maternal serum HCG and alpha fetal protein levels: Clinica significance and prediction of adverse pregnancy outcome. Int J Endocrinol Metab 11: 102-106, 2013.

7. Extermann P, Bischof P, Marguerat P and Mermillod B: Secondtrimester maternal serum screening for Down's syndrome: Free beta-human chorionic gonadotrophin (HCG) and alpha-fetoprotein, with or without unconjugated oestriol, compared with total HCG, alpha-fetoprotein and unconjugated oestriol. Hum Reprod 13: 220-223, 1998.

8. Sayin NC, Canda MT, Ahmet N, Arda S, Süt N and Varol FG: The association of triple-marker test results with adverse pregnancy outcomes in low-risk pregnancies with healthy newborns. Arch Gynecol Obstet 277: 47-53, 2008.
9. Shaw SW, Lin SY, Lin $\mathrm{CH}, \mathrm{Su} \mathrm{YN}$, Cheng PJ, Lee CN and Chen CP: Second-trimester maternal serum quadruple test for Down syndrome screening: A Taiwanese population-based study. Taiwan J Obstet Gynecol 49: 30-34, 2010.

10. Nix B, Wright $D$ and Baker A: The impact of bias in MoM values on patient risk and screening performance for Down syndrome. Prenat Diagn 27: 840-845, 2007.

11. Bishop J, Dunstan FD, Nix BJ and Reynolds TM: The effects of gestation dating on the calculation of patient specific risks in Down's syndrome screening. Ann Clin Biochem 32: 464-477, 1995.

12. Li Y, Zhang X, Sun Y, Hong D, Wang Y, Xu Z and Jiang T: Combined detection of $\alpha$-fetoprotein and free $\beta$-human chorionic gonadotropin in screening for trisomy 21 and management of cases in the moderate risk value range. Mol Clin Oncol 7: 623-628, 2017.

13. Parikh AM, Park AM and Sumfest J: Cumulative summation (CUSUM) charts in the monitoring of hypospadias outcomes: A tool for quality improvement initiative. J Pediatr Urol 10: 306-311, 2014.

14. Leandro G, Rolando N, Gallus G, Rolles K and Burroughs AK: Monitoring surgical and medical outcomes: The Bernoulli cumulative SUM chart. A novel application to assess clinical interventions. Postgrad Med J 81: 647-652, 2005. 